\title{
Effects of element or compound preexposure on taste-aversion learning with simultaneous and serial compounds
}

\author{
MATÍAS LÓPEZ RAMÍREZ \\ Universidad de Oviedo, Oviedo, Spain \\ and \\ LUIS AGUADO AGUILAR \\ Universidad Complutense de Madrid, Madrid, Spain
}

(Russell M. Church, Sponsor)

\begin{abstract}
Two taste-aversion learning experiments were done on the effects of preexposure to a flavor compound or to its elements on later conditioning to the compound. In Experiment 1, attenuation of latent inhibition was found after preexposing the separate elements only if the compound was simultaneous but not if it was formed by two flavors presented in sequence. In Experiment 2, the attenuation of latent inhibition by separate preexposure to the elements of a simultaneous compound was observed only with a short (4-h) and not with a long (48-h) preexposure-conditioning interval. These results are discussed in terms of generalization decrement between the compound and its elements and the flattening of generalization gradients with time.
\end{abstract}

It is well known that experience with a flavor before it is paired with illness as an unconditioned stimulus (US) reduces the aversion conditioned to that flavor, which is an example of the latent inhibition effect (Lubow, 1973). Latent inhibition is stimulus-specific, and its strength becomes reduced if the stimulus presented on the conditioning phase differs in some way from the preexposed stimulus (e.g., Siegel, 1969). A special case of stimulus change involves preexposing separately two different flavors and later conditioning them together, thus forming a compound. The results of this manipulation are interesting, because they bear on an important theoretical issue in conditioning theory-namely, whether a compound stimulus is best conceived as equivalent to the sum of its elements or as differing in some way from them (e.g., Kehoe \& Gormezano, 1980; Razran, 1971; Rescorla, 1973). This last conception predicts that a change from elements to compound between preexposure and conditioning should result in an attenuation of latent inhibition, owing to generalization decrement. In latent inhibition, this would mean that preexposure to the separate elements should generate less latent inhibition to the compound than preexposure to the compound itself. Contrary to these predictions, Holland and Forbes (1980) have reported that acquisition of an aversion to a simultaneous flavor compound was reduced more by preexposing the elements than by preexposing the compound. However, a recent attempt

Correspondence should be addressed to Luis Aguado Aguilar, Facultad de Psicología, Campus de Somosaguas, 28023 Madrid, Spain. by Baker, Haskins, and Hall (1990) failed to replicate this result in two experiments, and there is additional evidence from latent inhibition studies favoring the generalization decrement account (Honey \& Hall, 1988, 1989).

In the following two experiments, we studied two variables that might determine the effect of the change from elements to compound on latent inhibition. The first is the use of simultaneous versus serial compounds, in which one flavor is immediately followed by another (Experiment 1 ), and the second is the time interval between preexposure and conditioning (Experiment 2). The first variable might be important because simultaneous compounds of flavors are probably more easily discriminable from their constituents than serial compounds are from theirs. Mixing up both flavors might, for example, make the whole mix appear as a configuration, modify the way in which each flavor is perceived, or generate a new cue unique to that combination of flavors (Hull, 1943; Rescorla, 1973). If this is so, an attenuation of latent inhibition should be more probable with simultaneous than with serial compounds. The length of the interval between preexposure and conditioning is important, because of the positive relationship repeatedly found between generalization and the duration of the training-test interval.

\section{EXPERIMENT 1}

The aim of this experiment was to compare the effect of preexposure to the elements or the compound on later conditioning of simultaneous or serial compounds of two flavors. The design included six groups of subjects. Two 
groups (SIM-COMP and SER-COMP) were preexposed to a simultaneous or serial compound of two flavors, and another two groups (SIM-EL and SER-EL) were preexposed to the separate elements. Two additional groups (SER-CONT and SIM-CONT) acted as controls and did not receive any preexposure experience.

\section{Method}

Subjects and Apparatus. The subjects were 60 male Wistar rats. The rats were 140-150 days old at the beginning of the experiments, and their mean weight was $330 \mathrm{~g}$. The animals were housed in individual cages, with free food available. The solutions were presented in glass bottles with a stainless steel spout.

Vinegar and coffee were used as flavors, in solutions with concentrations of $1 \% \mathrm{v} / \mathrm{v}$ and $1 \% \mathrm{w} / \mathrm{v}$, respectively.

Procedure. The animals were progressively adapted to a water deprivation schedule, during which they had access to a bottle of water in daily periods of $10 \mathrm{~min}$ for 10 days. Both deprivation and the experimental procedures took place in the animals' home cages, placed in a dark room.

Preexposure. Subjects of Groups SIM-COMP, SER-COMP, SIM-EL, and SER-EL received two preexposure sessions, each with two trials. Group SIM-COMP received two 6-min exposures each day to a mix of coffee and vinegar. Group SER-COMP was exposed twice a day to a serial compound formed by a 3-min presentation of one of the elements, followed immediately by the alternative element presented for 3 additional minutes. Half the subjects received coffee as the first element, and the other half, vinegar. Groups SIM-EL and SER-EL received a daily 6-min exposure to each flavor, with order of presentation counterbalanced. During this phase, the subjects of the two control groups drank water for equivalent durations and at the same times. The interval between the two daily exposures to the flavors was $3 \mathrm{~h}$ in all cases. With the aim of equating groups in deprivation level, throughout the experiment all subjects drank water for $10 \mathrm{~min}, 3 \mathrm{~h}$ after the experimental sessions.

Conditioning and test. Conditioning took place on the day following the last preexposure session and consisted of a 6-min presentation of the simultaneous or the serial compound (3-min presentation of each flavor), followed $15 \mathrm{~min}$ later by an i.p. injection of $\mathrm{LiCl}(.15 \mathrm{M})$, with a proportion of $6-\mathrm{mg} / \mathrm{kg}$ body weight. On the following day, the animals received a recovery session, during which they had access to water for $10 \mathrm{~min}$ in their home cages. The test, given on the next day, consisted of a 6-min presentation of the corresponding compound in the experimental context.

\section{Results and Discussion}

Figure 1 shows mean consumption of the compound on the test day. The preexposed groups of the simultaneous and serial conditions drank more of the compound than did their respective control group, thus showing a latent

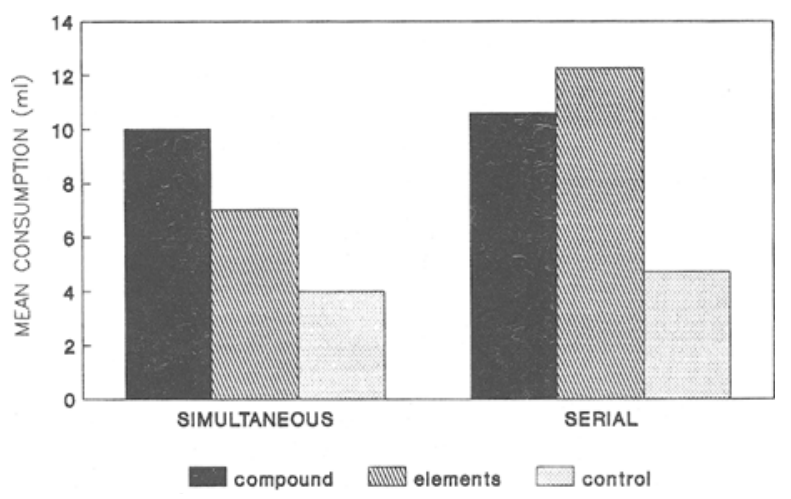

Figure 1. Mean consumption of the simultaneous and serial compounds on the test day in Experiment 1. inhibition effect. There were, however, differences between Groups SIM-COMP and SIM-EL, with higher consumption of the compound, and thus more latent inhibition, being shown by Group SIM-COMP. Mean consumption was $10.02,7.02$, and $4.16 \mathrm{ml}$ for Groups SIM-COMP, SIM-EL, and SIM-CONT, respectively.

Since there were no differences between consumption of the first element and consumption of the second element of the serial compound on the test day in any of the serial compound groups, the total amount of fluid consumed on this day is presented for these groups. Mean total consumption of fluid was $10.58,12.25$, and $4.74 \mathrm{ml}$ for Groups SER-COMP, SER-EL, and SER-CONT, respectively. An overall analysis of variance yielded significant effects of type of compound (simultaneous or serial) $[F(1,54)=10.29]$ and preexposure treatment $[F(2,54)=31.21]$, as well as a significant interaction $[F(2,54)=5.5$; all $p$ s $<.05]$. The relevant individual comparisons yielded the following results: for SIM-COMP versus SIM-EL, SIM-COMP versus SIM-CONT, and SIM-EL versus SIM-CONT, $F(1,54)=6.85,26.13$, and 6.22 , respectively (all $p s<.05$ ); for SER-COMP versus SER-EL, SER-COMP versus SER-CONT, and SER-EL versus SER-CONT, $F(1,54)=2.12(p>.05), 25.95$, and 42.92 (both $p s<.05$ ), respectively. As for the consumption of each flavor on the test phase by the serial preexposed groups, there were no differences between Groups SER-COMP and SER-EL for the first or the second element $[F(1,27)=1.302$ and 2.38 , respectively, $p>.05]$.

Although the results of the simultaneous compound groups failed to replicate Holland and Forbes's (1980) results, they do replicate those of Baker et al. (1990)that is, there was an attenuation of latent inhibition in the element-preexposed group-and they can be easily interpreted as a case of generalization decrement from the element preexposure phase to the compound conditioning phase. However, an equivalent comparison with serial compounds showed a similar latent inhibition effect in both preexposed groups and thus no generalization decrement from preexposure to conditioning. These results suggest that only when simultaneous compounds are used does switching from elements to compound between the preexposure and conditioning phases produce a generalization decrement that results in an attenuation of latent inhibition.

From this, it may be inferred that although the animals discriminate between a simultaneous flavor compound and its elements, they treat a serial compound as functionally equivalent to its separate elements. At least in relation to the processes that underlie the latent inhibition effect, serial compounds of flavors do not seem to have properties different from those of their elements, and there is no need to appeal to any feature that emerges as a result of the sequential pairing of these stimuli.

\section{EXPERIMENT 2}

The results of former experiments showing increased generalization with increasing training-test intervals in 
several learning tasks (McAllister \& McAllister, 1963; Perkins \& Weyant, 1958; Thomas et al., 1985) lead to the expectation that extending the interval between preexposure and compound conditioning in a latent inhibition experiment should tend to increase generalization of the effects of preexposure, thus producing an equivalent latent inhibition effect, no matter whether the compound or the elements have been preexposed. In Experiment 2, we tested this hypothesis by preexposing the animals to a simultaneous compound or to its elements and conditioning the compound after two different time intervals.

\section{Method}

Subjects. Subjects were 50 male Wistar rats, with a mean weight of $302 \mathrm{~g}$ at the start of the experiment and without prior experimental experience.

Procedure. Deprivation, housing conditions, concentrations of the flavors, and $\mathrm{LiCl}$ dose were similar to those in Experiment 1 .

Preexposure. The subjects were divided into four groups differing in preexposure procedure (elements, compound, or no preexposure) and duration of the interval between preexposure and conditioning (4 or $48 \mathrm{~h}$ ). All preexposed groups were exposed for an equivalent amount of time to coffee and vinegar, in an attempt to control for the potentially confounding effect of the different total times during which the animals of Groups SIM-COMP and SIM-EL of Experiment 1 were exposed to the flavors (24 and $12 \mathrm{~min}$, respectively). Groups COMP-48 and COMP-4 were given two 5-min preexposure trials with a simultaneous compound of coffee and vinegar. Animals of Groups COMP-48 and COMP-4 were given water for $5 \mathrm{~min}$ after each preexposure trial, so that all groups were equated in their opportunity to drink. Groups EL-48 and EL-4 were exposed once to the solutions of coffee and vinegar, each presented separately for a period of $10 \mathrm{~min}$, with order of presentation counterbalanced. Animals in the control group (CONT) drank water during two 10-min periods. The two presentations of the fluids during the preexposure phase were separated by a 5-h interval in all groups.

Conditioning and test. Conditioning consisted of the presentation of the compound for $10 \mathrm{~min}$, followed $15 \mathrm{~min}$ later by an i.p. injection of $\mathrm{LiCl}$. This phase took place 4 or $48 \mathrm{~h}$ after the last preexposure trial in Groups COMP-4 and EL-4 and Groups COMP-48 and EL-48, respectively. The animals of the 48 -h interval groups received water during $10 \mathrm{~min}$ in their home cages on the day intervening between the preexposure and the conditioning phases. On each of the 2 test days, all animals were given access to the compound for a period of $10 \mathrm{~min}$. Two hours after the first test trial, the animals received water for an additional period of $10 \mathrm{~min}$.

\section{Results}

Figure 2 shows consumption of the compound by the different groups, averaged over the 2 test days. Mean consumption of the compound was $3.71,5.92,3.09,3.5$, and $1.46 \mathrm{ml}$ for Groups COMP-48, COMP-4, EL-48, and EL-4, respectively. All preexposed groups showed higher consumption than did the control group, thus showing a reduced aversion to the compound relative to the control group. However, although both preexposed groups showed a similar aversion to the compound when the interval between preexposure and conditioning was $48 \mathrm{~h}$, the animals preexposed to the compound showed more consumption than did those preexposed to the elements when that interval was only $4 \mathrm{~h}$. Thus, latent inhibition was attenuated after element preexposure only with a 48 -h preexposureconditioning interval.

An analysis of variance performed on the consumption data from the 2 test days yielded a significant effect both

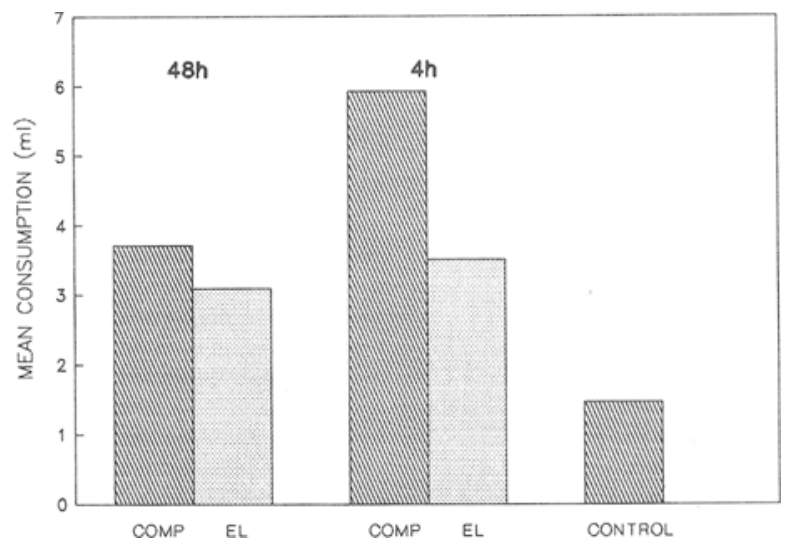

Figure 2. Mean consumption of the compound on the test day in Experiment 2.

of the preexposure procedure $[F(2,44)=15.74, p<.05]$ and of the preexposure-conditioning interval $[F(1,44)=$ 5.53 , ps $<.05$ ], but there was no significant interaction of these factors $[F(1,44)=1.24, p>.05]$. Individual comparisons showed significant differences between each preexposed group and the control group: for COMP-48 versus CONT, EL-48 versus CONT, COMP-4 versus CONT, and EL-4 versus CONT, $F(1,44)=4.16,5.2$, 20.29 , and 29.46, respectively (all ps $<.05$ ). Moreover, individual comparisons between the groups preexposed to the compound or to the elements showed significant differences with a 4 -h preexposure-conditioning interval but not with a 48-h interval [for COMP-4 vs. EL-4, $F(1,44)$ $=10.87, p<.05$; for COMP-48 vs. EL-48, $F(1,44)=$ $0.70, p<.05]$.

\section{GENERAL DISCUSSION}

The attenuated latent inhibition to a simultaneous compound observed in Experiment 1 after element preexposure, together with an equivalent result reported by Baker et al. (1990), stands in clear contrast to the results of Holland and Forbes (1980), who instead found stronger latent inhibition after element preexposure, and it can be easily interpreted as a case of generalization decrement. A new finding from our Experiment 1, however, was that latent inhibition to a serial compound was equivalent after element or compound preexposure, suggesting that changing from elements to compounds does not produce generalization decrement with serial compounds. This result is contrary to the findings of Kaye, Swietalsky, and Mackintosh (1988), who observed an attenuation of latent inhibition with the use of serial compounds (two flavors separated by a 5-min interval), whether the target element was preexposed and later conditioned followed by a new flavor or this serial compound was preexposed and the target conditioned alone. Kaye et al. suggested that the subjects might have perceived the succession of the two flavors as a configuration, so that there could be a generalization decrement between this configuration and the separate elements. However, our results show that even when the elements are presented in strict succession, which should presumably maximize the probability of the compound's being perceived as a configuration or of any other kind of interaction between its elements, there was no evidence of generalization decrement.

The absence of differences between the effects of element or compound preexposure with serial compounds also raises problems for some theories of latent inhibition that would predict such differences. For example, Lubow's conditioned attention theory (Lubow, 1989; Lubow, Schnur, \& 
Rifkin, 1976) states that preexposure to a stimulus, S1, followed by a second stimulus, S2, prevents S1 from losing associability due to conditioning of attentional responses to it. The theory predicts that preexposure to $S 1$ followed by $\mathbf{S} 2$ should attenuate latent inhibition, relative to separate exposure to S1. Wagner's (1981) latent inhibition theory also predicts that preexposure to the S1-S2 sequence should attenuate latent inhibition to $S 1$, due in this case to $S 2$ 's interfering with the processing of S1.

It is a well-known fact that generalization gradients tend to flatten with time (e.g., Perkins \& Weyant, 1958), and some researchers have interpreted this fact as reflecting a process of forgetting in long-term memory (Thomas, 1981). As the interval between conditioning and the generalization test increases, the precision of the memory trace is reduced, so that the animal tends to confuse the test stimuli with the original one. The effect of the length of the preexposure-conditioning interval observed in our Experiment 2 may be taken as a new manifestation of this phenomenon.

\section{REFERENCES}

Baker, A. G., Haskins, C. E., \& Hall, G. (1990). Stimulus generalization decrement in latent inhibition to a compound following exposure to the elements or the compound. Animal Learning \& Behavior, 18, 162-170.

Holland, P. C., \& Forbes, D. T. (1980). Effects of compound or element preexposure on compound flavor aversion conditioning. Animal Learning \& Behavior, 8, 199-203.

Honey, R. C., HALL, G. (1988). Overshadowing and blocking procedures in latent inhibition. Quarterly Journal of Experimental Psychology, 40B, 163-186.

HoNEY, R. C., HALL, G. (1989). Attenuation of latent inhibition after compound pre-exposure: Associative and perceptual explanations. Quarterly Journal of Experimental Psychology, 41B, 355-368.

Hull, C. L. (1943). Principles of behavior. New York: AppletonCentury-Crofts.

Kaye, H., Swietalski, N., \& Mackintosh, N. J. (1988). Distractor effects on latent inhibition are a consequence of generalization decrement. Quarterly Journal of Experimental Psychology, 40B, 151-161.
Kehoe, E. J., \& Gormezano, I. (1980). Configuration and combination laws in conditioning with compound stimuli. Psychological Bulletin, 87, 351-378.

Lubow, R. E. (1973). Latent inhibition. Psychological Bulletin, 79, 398-407.

LuBow, R. E. (1989). Latent inhibition and conditioned attention theory. Cambridge: Cambridge University Press.

Lubow, R. E., SchnuR, P., RifkIN, B. (1976). Latent inhibition and conditioned attention theory. Journal of Experimental Psychology: Animal Behavior Processes, 2, 163-174.

MCAllister, W. R., MCAllister, D. E. (1963). Increase over time in the stimulus generalization of acquired fear. Journal of Experimental Psychology, 65, 576-582.

Perkins, C. C., \& Weyant, R. G. (1958). The interval between training and test trial as a determiner of the slope of generalization gradients. Journal of Comparative \& Physiological Psychology, 51, 596-600.

RAzRAN, G. (1971). Mind in evolution. New York: Appleton-CenturyCrofts.

Rescorla, R. A. (1973). Evidence for the "unique stimulus" account of configural conditioning. Journal of Comparative \& Physiological Psychology, 85, 331-338.

SIEGEL, S. (1969). Generalization of latent inhibition. Journal of Comparative \& Physiological Psychology, 69, 157-159.

Thomas, D. R. (1981). Studies of long-term memory in the pigeon. In N. E. Spear \& R. R. Miller (Eds.), Information processing in animals: Memory mechanisms (pp. 257-290). Hillsdale, NJ: Erlbaum.

Thomas, D. R., Windell, B. T., BakKe, I., KreYe, J., Kimose, E., aposhyAN, H. (1985). Long-term memory in pigeons: I. The role of discrimination problem difficulty assessed by reacquisition measures. II. The role of stimulus modality assessed by generalization slope. Learning \& Motivation, 16, 464-477.

WAGNER, A. R. (1981). SOP: A model of automatic memory processing in animal behavior. In N. E. Spear \& R. R. Miller (Eds.), Information processing in animals: Memory mechanisms (pp. 5-47). Hillsdale, NJ: Erlbaum.

(Manuscript received February 3, 1992.) 